\title{
Looking for the elusive 3:2 ratio of high-frequency quasi-periodic oscillations in the microquasar XTE J1550-564
}

\author{
Peggy Varniere \\ APC, AstroParticule et Cosmologie, Université Paris Diderot, CNRS/IN2P3, CEA/Irfu, \\ Observatoire de Paris, Sorbonne Paris Cité, 10, rue Alice Domon et Lonie Duquet, 75205 \\ Paris Cedex 13, France \\ AIM, CEA, CNRS, Universit Paris-Saclay, Université Paris Diderot, Sorbonne Paris Cit, \\ F-91191 Gif-sur-Yvette, France. \\ and \\ Jérôme Rodriguez \\ AIM, CEA, CNRS, Universit Paris-Saclay, Université Paris Diderot, Sorbonne Paris Cit, \\ F-91191 Gif-sur-Yvette, France. \\ varniere@apc.univ-paris7.fr, jrodriguez@cea.fr \\ Received / Accepted
}

Received —_ accepted 


\begin{abstract}
Using the two main XTE J1550-564 outbursts (1998-99 and 2000) we gathered about 30 observations with confirmed detections of HFQPOs. While this is a small sample it is enough to start looking at the generic properties of these oscillations, especially focusing on their frequencies and their potential harmonic relationship. This then will provide us with a list of constraints, necessary for any attempt of modelling their origin. We defined five groups based on their similarities in the Fourier domain, namely the continuum of their power density spectra (PDS) and the HFQPO frequencies. We then combined the individual PDSs of each family to obtain a PDS with higher statistics to search for other potential, previously undetected, weaker peaks. While we have two $3 \sigma$ potential detections of a pair of HFQPOs in our combined PDSs, none of them shows HFQPOs with frequencies in a previously claimed 3:2 ratio. Using the results presented here we propose an updated list of requirements for any model trying to explain the HFQPOs in microquasars.
\end{abstract}

Subject headings: accretion, accretion disks- black hole physics- stars: individual (XTE J1550-564)- stars: oscillations 


\section{Introduction}

Since their first detection there have been a long string of efforts to understand the source of the variability observed in microquasars but no model has yet gained wide acceptance. This is especially true for the origin of the rather elusive High-Frequency Quasi-Periodic Oscillation (HFQPO) in systems containing (or thought to contain) a black hole $(\mathrm{BH})$ as compact object. HFQPOs appear as narrow peak(s) in the X-ray power-density spectra (PDS) of BH binaries. They have been detected in eight different BH sources (GRO J1655-40, GRS 1915+105, XTE J1550-564, H1743-322, 4U 1630-47, XTE J1650-500, XTE J1859+226 IGR J17091-3624, e.g. Remillard \& McClintock 2006; Altamirano \& Belloni 2012; Belloni et al. 2012, and references therein), ranging from as low as $27 \mathrm{~Hz}$ in GRS 1915+105 (Belloni et al. 2001) up to a few hundred $\mathrm{Hz}$ (a maximum of $450 \mathrm{~Hz}$ was seen in GRO J1655-40 Remillard \& McClintock 2006). HFQPOs are particularly interesting as their frequencies typically lie in the frequency range of the Keplerian frequency of the last stable orbit around the central BH. They can therefore be seen as a window to the innermost region of accretion where strong gravity is expected to play an important role ${ }^{1}$. This is one of the reasons why HFQPOs have stimulated much more interest than their low frequency counterparts (LFQPO) even though they are much weaker and rarer.

Somes sources, and especially XTE J1550-564, have exhibited HFQPOs with enough regularity to start studying their properties with respect to the source's spectral states and also search for groups (or families) of similar PDSs that can then be combined in order to improve the statistics and probe fainter components. Here we aim at presenting

\footnotetext{
${ }^{1}$ Relativistic effects, and their influences on the properties of low frequency QPOs, are, for example, discussed within the context of the accretion-ejection instability in Varnière et al. (2012).
} 
a comprehensive study of the occurrences of HFQPOs in the outburst of XTE J1550-564. While most of the data are taken from published papers (Remillard et al. 2002a; Miller et al. 2001; Sobczak et al. 2000a; Rodriguez et al. 2003; Belloni et al. 2012), we reanalyzed and combined some observations together to try and obtain better statistical significances on the presence and obtained parameters of already known XTE J1550-564's HFQPOs. Using the combined PDSs we then study the possible simultaneous presence of multiple high frequency peaks (or pairs of HFQPOs), and if present investigate the ratio of their frequencies. Using five groups of observations we can then see how the presence/absence of an additional peak improves, and increases the constraints already known on models of HFQPOs. We then conclude this paper with a short list of constraints that any model trying to explain HFQPOs should respect.

\section{Looking for the elusive 2:3 relationship between the HFQPOs frequencies}

Many authors have pointed out a ratio 3:2 between different frequencies of HFQPOs in various sources such as XTE J1550-564 (Miller et al. 2001) and GRO J1655-40 (Remillard et al. 2002a). Such pairs are, however, not always detected, and in some reported cases they can show different frequency ratios than the 3:2 e.g. Strohmayer (2001) and Belloni \& Altamirano (2013) in the case of GRS 1915+105. Since the predicted systematic presence of a specific ratio, such as the 3:2 would be a strong constraint on theoretical models we decided to explore this aspect in more detail. In XTE J1550-564 HFQPOs have been detected at various frequencies (from $\sim 100 \mathrm{~Hz}$ to $\sim 280 \mathrm{~Hz}$ ) both during the major 1998-1999, and fainter 2000 outbursts (Remillard et al. 2002a; Miller et al. 2001). Belloni et al. (2012) analyse the properties of HFQPOs of various BH sources. In addition to HFQPOs reported earlier (for which they obtain compatible frequencies), they also report new occurrences of HFQPOs when analysing only the hard $(\gtrsim 6 \mathrm{keV})$ band light curves in 
four additional observations. Pairs of HFQPOs have, however, not been detected in any of the individual observations considered in these studies, but they were reported from stacked observations, as we describe below.

In the upper panel of Fig,1 we report the distribution of frequencies for all HFQPOs detected in XTE J1550-564 (Remillard et al. 2002a; Miller et al. 2001) as function of the ratio of the disk flux to the total flux as obtained from spectral fittings (Sobczak et al. 2000a; Rodriguez et al. 2003). When looking purely on the frequency level, the distribution exhibits two broad 'clusters', that may be in the 3:2 ratio, although the large variations of frequencies between the different observations, or even within a given cluster, prevent any firm conclusion to be obtained. Miller et al. (2001) produced a global PDS from all observations from the 2000 outburst showing HFQPOs. This total PDS exhibited a pair of HFQPOs, with a main feature at $268 \pm 3 \mathrm{~Hz}$ and a a fainter one at $188 \pm 3 \mathrm{~Hz}$ (Miller et al. 2001, Fig. 1 lower panel). Remillard et al. (2002a) separated the observations of the 1998/1999 outburst according to the type of LFQPO they detected. They note an apparent anti correlation between the amplitude of the HF and LFQPO. They also report that broad $\sim 180 \mathrm{~Hz}$ QPOs are usually associated with type B LFQPOs, while narrow $\sim 280 \mathrm{~Hz}$ QPO are associated with broad type A LFQPO. None of the observations they analyzed showed the presence of a pair of HFQPOs when analyzed alone. However, accumulating all the (HFQPO) observations with type B LFQPOs on the one hand and those with type A LFQPOs on the other hand, Remillard et al. (2002a) found that each of the two resultant PDSs exhibited a pair of HFQPOs, one close to $276 \mathrm{~Hz}$ (respectively 281 and $277 \mathrm{~Hz}$ ) and the other close to $184 \mathrm{~Hz}$ (respectively 187 and $185 \mathrm{~Hz}$ ). Here again the proximity of the frequency ratios with 2:3 led to the conjecture that the HFQPOs are naturally occurring in a 2:3 ratio, a statement further strengthened by the detection, in one case, of the possible fundamental (or the "1" of a 1:2:3 ratio at $92 \mathrm{~Hz}$ (Remillard et al. 2002a). 

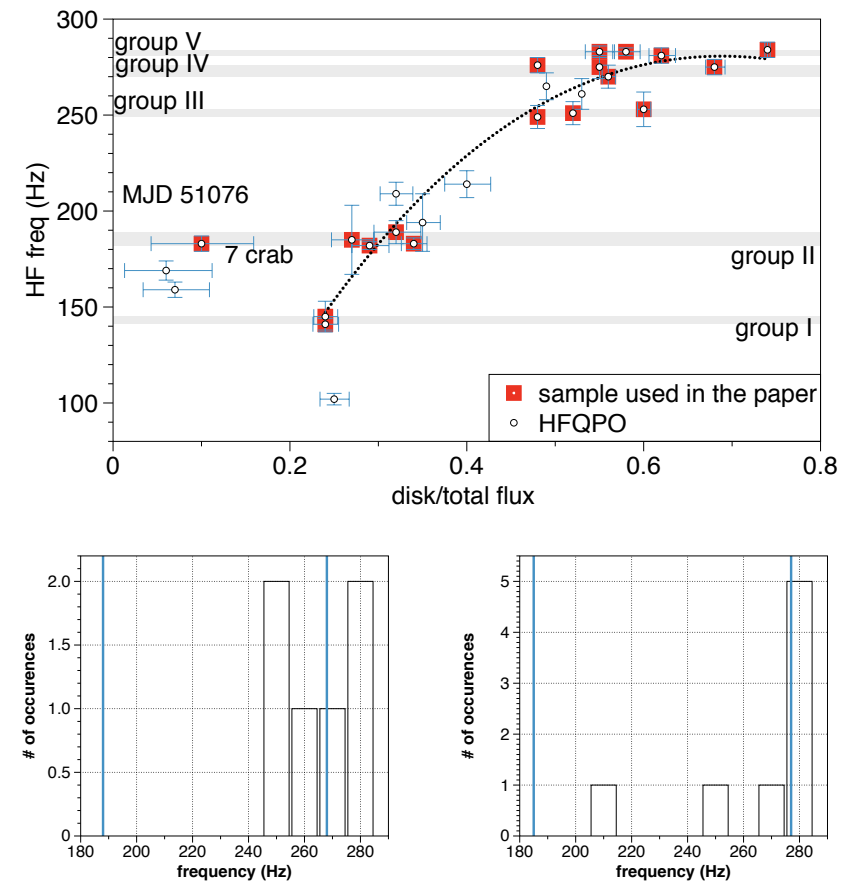

Fig. 1.- Upper panel: distribution of the HFQPOs' frequencies in XTE J1550-564 during the two outbursts of 1998-99 and 2000 as function of the ratio of the disk flux to the total flux. The horizontal grey bands represent the groups of HFQPOs we explore in this paper. Lower panels: Distribution of the HFQPOs' frequencies in individual observations that were added together by Miller et al. (2001) on the left and Remillard et al. (2002a) on the right. The vertical line shows the frequency detected in the averaged PSD. 
As one can see in the lower panel of Fig, 1, the discovery of this ratio was based on adding together many observations with a HFQPO showing a rather wide range of frequencies. This renders the genuineness and interpretation of this 3:2 ratio difficult as some frequencies are present in individual observations but not in the averaged data, while some other reach detectability. One way to overcome the non-detectability by adding more statistics without adding more unknowns is to focus on observations where 1) the already detected frequencies are very close and 2) the continuum of the PDS, and the LFQPO complex are also similar. Having a similar continuum means that the behavior/parameters of the inner accretion flow is basically the same and will not influence the PDS by inducing the appearance of extra/unrelated components. This is especially relevant as we showed that a microquasar's states could be defined by the presence/absence of LF and/or HF QPOs and that these states could be interpreted within a sound theoretical framework (Varnière et al. 2011). Said with different words we avoid to include other sources of variabilities than the one common to all the observations considered together that could impact the PDS in an unknown manner. For the same reason, restraining ourselves to add observations harboring similar HFQPO frequencies will allow us to ensure that the system's parameters are very similar, and that any other frequencies detected in the averaged PDS is genuinely related to an improved statistics.

When looking at Fig. 1 (upper panel), we see that there seems to be several groups of HFQPOs with close frequencies (i.e. within a few $\mathrm{Hz}$ from one another; this permits us to define five groups, see Sec. 2.1, that are represented by the grey horizontal shading in Fig. 11. Using this we will try to answer the following questions: 1) do we detect a fainter HFQPO when combining several observations showing similar PDSs and HFQPOs and in this case 2) how those fainter HFQPOs relate to the already detected one. With those answers we will be able to probe an eventual relation between the peaks, and in particular check the robustness of the $3: 2(: 1)$ ratio. 


\subsection{RXTE observations and data reduction}

We re-analysed all RXTE observations where a HFQPO was reported (Miller et al. 2001; Remillard et al. 2002b; Belloni et al. 2012), and further selected those showing multiple (at least two) occurrences of the supposedly same HFQPO. Those are shown as squares (hollow red in the online version) in Fig.1. We considered HFQPOs reported from different observations as being the same when the difference of their centroid frequencies did not exceed $5 \mathrm{~Hz}$. Of the $\sim 35$ reports of HFQPOs we then determine five families with distinct frequencies: $\sim 141 \mathrm{~Hz}$, a $\sim 183 \mathrm{~Hz}$, a $\sim 250 \mathrm{~Hz}, \mathrm{a} \sim 275 \mathrm{~Hz}$, and a $\sim 283 \mathrm{~Hz} \mathrm{HFQPO}$, defining what is hereafter referred to as a 'group' of data. In order to try and increase even more the statistics of our groups, we also considered the $R X T E$ observations found at less than 0.5 day from those where HFQPOs were reported if they have a similar continuum, even if they do not show evidence for the presence of a high frequency feature. Note that Belloni et al. (2012) also reports the detection of HFQPOs near $263 \mathrm{~Hz}$ in two observations of the 2000 outburst. These features were, however, only detected from the analysis of hard $(>6 \mathrm{keV}$ ) light curves, and since the sample is small (amounting a total of $4 \mathrm{ks}$ of data) we decided not include them here ${ }^{2}$, Remillard et al. (2002b) also report two occurrences of an HFQPO near $210 \mathrm{~Hz}$. Inspection of the individual PDSs show that they are quite different, and therefore we did not consider them as defining a group. The journal of the observations analyzed in this paper is reported in table 2.1 .

The data from the $R X T E /$ proportional counter array (PCA) were reduced with the HEASOFT v6.17 suite following standard procedures to define the good time intervals and obtain light curves filtered from data taken at low elevations above the Earth,

\footnotetext{
${ }^{2}$ We nevertheless produced a 'hard' PDS from these two observations, and apart from the already reported $263 \mathrm{~Hz}$ QPO (Belloni et al. 2012), we do not see anything special in this PDS.
} 
and large offset from the source. For each observation, we produced a high resolution $\left(2^{-12} \mathrm{~S}=244.140625 \mu \mathrm{s}\right)$ light curve covering the full PCA energy range by combining Single Binned and Event data modes. These individual light curves were then fed to POWSPEC to produce single-observation power density spectra (PDS, hereafter individual PDS), which were obtained by averaging "sub"-PDSs calculated over temporal intervals of 4 s long. The resultant individual PDSs were, then, rebinned geometrically. As the source can be very bright (up to $\sim 13000 \mathrm{cts} / \mathrm{s} / \mathrm{PCU}$ ) dead time affects the count rates, and in particular artificially lowers the level of Poisson noise in the PDSs. The dead time corrected Poisson noise $P N(f)$ was estimated for each individual PDS by fitting the high frequency (> 500 $\mathrm{Hz}$ ) part of the PDS with a constant. Each individual PDS was then corrected from $P N(f)$, and all white noise corrected individual PDSs belonging to a given group were averaged following

$$
\mathrm{PDS}_{\text {group }}=\frac{\sum_{i} T_{i} \times \mathrm{PDS}_{i}}{\sum_{i} T_{i}}
$$

with group $\subset$ [I, II, III, IV, V], $\mathrm{PDS}_{i}$ the individual PDS of observation $i$, and $T_{i}$ the total exposure of observation $i$.

In all cases the global PDSs have been fitted with XSPEC V12.9.0i. We first explored the $0.25-1000 \mathrm{~Hz}$ PDS, and fitted the full band PDS. In all cases two or three zero-centered broad Lorentzians were used to model the PDSs' continuum. Narrower Lorentzians (i.e. where $\mathrm{Q}=\nu_{\mathrm{QPO}} / \mathrm{FWHM} \mathrm{QPO}_{\mathrm{Q}} \gtrsim 2$ ) were then included to represent the low and high frequency QPOs until a satisfactory fit was obtained. All errors are given at the $90 \%$ confidence level. To estimate the properties of the HFQPO, we, then, omitted the frequencies below $50 \mathrm{~Hz}$ (see also the discussion in Belloni et al. 2012). The fit to the continuum is much cleaner and does not depend on a proper modelling of the low frequency structures. Simpler models for the continuum were used in this second approach. We verified that the parameters obtained for the HFQPOs were compatible with both approaches. As this was the case for 
ObsId Date Rate $^{a}$ Exposure HFQPO

\begin{tabular}{|c|c|c|c|c|}
\hline \multicolumn{3}{|c|}{ (MJD) } & $(\mathrm{s})$ & $(\mathrm{Hz})$ \\
\hline \multicolumn{5}{|c|}{ Group I: $\sim 141 \mathrm{~Hz}$ feature ${ }^{\dagger}$} \\
\hline 30191-01-31-00 & 51101.61 & 3107 & 1434 & $141 \pm 3$ \\
\hline 30191-01-31-01 & 51101.94 & 3058 & 2019 & $145 \pm 8$ \\
\hline total exposure & & & 3453 & \\
\hline \multicolumn{5}{|c|}{ Group II: $\sim 183 \mathrm{~Hz}$ feature ${ }^{\dagger}$} \\
\hline $30191-01-02-00$ & 51076.00 & 13223 & 2966 & $183 \pm 4$ \\
\hline 30191-01-33-00 & 51108.08 & 3575 & 9450 & $183 \pm 3$ \\
\hline $40401-01-53-00$ & 51245.36 & 4607 & 2288 & $182 \pm 2$ \\
\hline $40401-01-55-00$ & 51247.98 & 4439 & 2462 & $189 \pm 6$ \\
\hline $40401-01-56-00$ & 51249.40 & 4131 & 542 & $185 \pm 18$ \\
\hline 40401-01-56-01 & 51249.47 & 4084 & 180 & $185 \pm 18$ \\
\hline total exposure & & & $17888 \mathrm{~s}$ & \\
\hline
\end{tabular}

Group III: $\sim 250 \mathrm{~Hz}$ feature

\begin{tabular}{llccl}
\hline $40401-01-71-00$ & 51270.74 & 733 & 3448 & $253 \pm 9^{\dagger}$ \\
$40401-01-72-00$ & 51271.41 & 705 & 1976 & \\
$50134-02-05-00$ & 51669.19 & 958 & 2078 & $249 \pm 6^{\ddagger}$ \\
$50134-02-08-00$ & 51672.41 & 801 & 1599 & $251 \pm 6^{\ddagger}$ \\
$50134-02-08-01$ & 51672.96 & 1025 & 1536 & \\
$50134-01-01-00$ & 51673.40 & 962 & 1594 & \\
\hline total exposure & & & $12231 \mathrm{~s}$ \\
\hline
\end{tabular}

$a$ in units of counts/s per PCU on

$\dagger$ Report and frequencies of HFQPO from Remillard et al. (2002b).

$\ddagger$ Report and frequencies of HFQPO from Miller et al. (2001).

* Report and frequencies of HFQPO from Belloni et al. (2012), from a hard ( $\gtrsim 6 \mathrm{keV})$ band only. 
ObsId Date Rate $^{a}$ Exposure HFQPO

\begin{tabular}{|c|c|c|c|c|}
\hline \multicolumn{3}{|c|}{ (MJD) } & $(\mathrm{s})$ & $(\mathrm{Hz})$ \\
\hline \multicolumn{5}{|c|}{ Group IV: $\sim 275 \mathrm{~Hz}$ feature } \\
\hline 30191-01-36-00 & 51115.28 & 2830 & 2070 & $270 \pm 6^{\dagger}$ \\
\hline 40401-01-61-00 & 51258.09 & 1927 & 800 & $275 \pm 4^{\dagger}$ \\
\hline $40401-01-61-01$ & 51258.50 & 1882 & 1116 & $276_{-6}^{+7 \star}$ \\
\hline $40401-01-62-00$ & 51258.97 & 1812 & 1711 & \\
\hline $40401-01-62-01$ & 51259.25 & 1772 & 735 & $275 \pm 4^{\star}$ \\
\hline $50134-02-02-01$ & 51664.41 & 1500 & 2253 & $276 \pm 4^{\ddagger}$ \\
\hline $50134-02-03-00$ & 51664.64 & 1581 & 2870 & $275 \pm 4^{\ddagger}$ \\
\hline total exposure & & & $11555 \mathrm{~s}$ & \\
\hline \multicolumn{5}{|c|}{ Group V: $\sim 283 \mathrm{~Hz}$ feature } \\
\hline 30191-01-41-00 & 51126.59 & 678 & 4120 & $284 \pm 4^{\dagger}$ \\
\hline 40401-01-50-00 & 51241.80 & 4199 & 3120 & $283 \pm 2^{\dagger}$ \\
\hline $40401-01-51-00$ & 51242.51 & 4049 & 1517 & $283 \pm 3^{\dagger}$ \\
\hline 40401-01-59-01 & 51255.09 & 2402 & 983 & $281 \pm 4^{\dagger}$ \\
\hline 40401-01-59-00 & 51255.16 & 2369 & 3722 & $281 \pm 2^{\star}$ \\
\hline total exposure & & & $13462 \mathrm{~s}$ & \\
\hline
\end{tabular}

$a$ in units of counts/s per PCU on

$\dagger$ Report and frequencies of HFQPO from Remillard et al. (2002b).

$\ddagger$ Report and frequencies of HFQPO from Miller et al. (2001).

* Report and frequencies of HFQPO from Belloni et al. (2012), from a hard ( $~ 6 \mathrm{keV})$ band only. 
all groups, and since our focus here is on the HFQPO, we present only the results obtained with the simplest/cleanest approach.

The choice to white noise correct the observations individually first was dictated by the fact that they can have very different intensities, and, therefore, can be affected differently by dead time effects (in other words $\mathrm{PN}(\mathrm{f})$ is observation-dependent). We, however, remark that, in general, for studies of HFQPOs, the level of white noise is not subtracted from the PDS, but fitted together with the other components (e.g. Miller et al. 2001; Belloni et al. 2012). In addition, in Belloni et al. (2012) PN(f) was not consider as constant and was fitted with a power law model with free parameters. To check the consistency of our method and robustness of the results obtained, we also followed a procedure where all individual, non-PN corrected PDS were averaged following Eq. 1, and fitted all components in the group PDS thus obtained. PN(f) was fitted with a power law with free parameters (Belloni et al. 2012). We followed two approaches:

1. We fitted each group PDS over the full frequency range (therefore including the low frequency features), and then kept the best obtained model to estimate the parameters of the HFQPO(s), focusing on the frequency range above $50 \mathrm{~Hz}$.

2. We only considered the frequency range above $50 \mathrm{~Hz}$ (ignoring thus the low frequency complex), to evaluate the continuum (including $\mathrm{PN}(\mathrm{f})$ ) and the parameters of the $\operatorname{HFQPO}(\mathrm{s})$.

In all cases, PN(f) has a very low power law index (of the order of $10^{-3}$ and sometimes compatible with zero). The parameters of the HFQPOs obtained with these two methods are very close (if not identical), and nevertheless always compatible within the (90\%) errors, to the one obtained with our method, showing the compatibilities of the different 
approaches. The rest of the paper, therefore, presents and discusses the results obtained with our method.

\subsection{Group I: HFQPO at $\sim 141 \mathrm{~Hz}$}

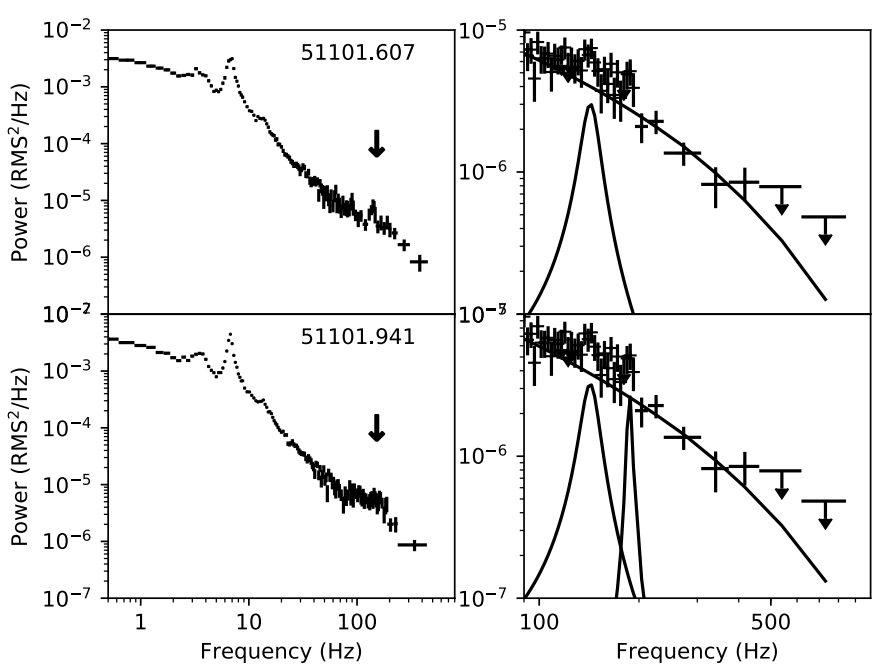

Fig. 2.- Left: Individual PDSs of the two observations of group I with a potential $140 \mathrm{~Hz}$ HFQPO. The vertical arrow shows the approximate location of the HFQPO, which is rather clear in the upper panel.Right: Zoom on the combined PDS from the two observations. The continuous lines represents the individual components of the fit, here a cut-off powerlaw for the continuum, and either one (upper panel) or two (lower panel) Lorentzian lines at $141 \mathrm{~Hz}$ and $187 \mathrm{~Hz}$.

Figure 2 (left) shows the individual PDSs of the two observations considered for the analysis of the $\sim 140 \mathrm{~Hz}$ feature. The HF feature is more obvious in the first observation, and was reported both in Remillard et al. (2002b) and Belloni et al. (2012), while the second is only mentioned in the former publication.

When omitting the frequencies below $50 \mathrm{~Hz}$, a zero-centered Lorentzian represents the overall continuum of the combined PDS pretty well $\left(\chi_{\nu}^{2}=1.4,61\right.$ degrees of freedom, 
dof). Some residuals are visible in the the region around $140 \mathrm{~Hz}$, close to frequency of the previously claimed HFQPOs (Remillard et al. 2002b; Belloni et al. 2012, Table 2.1). The inclusion of a Lorentzian improves the fit with $\Delta \chi^{2}=20.3$ for three additional free parameters. The width of the feature is not well constrained, and may tend to very broad values. It was frozen to the value reported by Belloni et al. (2012), still providing a good fit $\left(\chi_{\nu}^{2}=1.1\right.$ for 59 dof $)$. This frozen-width HFQPO is significant at a level of $4.2 \sigma$. Interestingly some excess is still visible around 180-200 Hz (Fig. 2). Adding another Lorentzian, however, provides a marginal improvement to the fit $\left(\Delta \chi^{2} \sim 10\right.$ for three additional parameters). The additional component is, indeed, statistically non-significant $(1.7 \sigma)$, and we estimate a $3 \sigma$ upper limit of about 1\% for a Q 10 HFQPO at this frequency. We do not consider it further in this study, and take the upper limit estimated here as a proxy for the limit of an additional HFQPO for this group.

\subsection{Group II: HFQPO at $\sim 183 \mathrm{~Hz}$}

Figure 3 (left) shows the PDSs of all observations considered for the analysis of the $\sim 183 \mathrm{~Hz}$ feature. We see significant differences in the shapes of the (first and five following) PDSs and in the presence and values of the centroid frequencies of the main LFQPO Remillard et al. 2002b, indicate variations between 5.4 and $6.4 \mathrm{~Hz}$ for the 5 similar PDSs, and the LFQPO frequency can go up to $13.2 \mathrm{~Hz}$ if we consider the first observation). The LFQPOs are classified as type B by Remillard et al. (2002b), although those of MJD 51108-51249 show a continuum made of "flat-top" noise at low frequencies, and rather strong LFQPO complexes with (sub)-harmonics that are usually reminiscent of type Cs. Despite the different shapes and LFQPO frequencies at low frequencies, we averaged all PDSs of group II together to produce the PDS shown in Fig. 3 (right), especially to take 

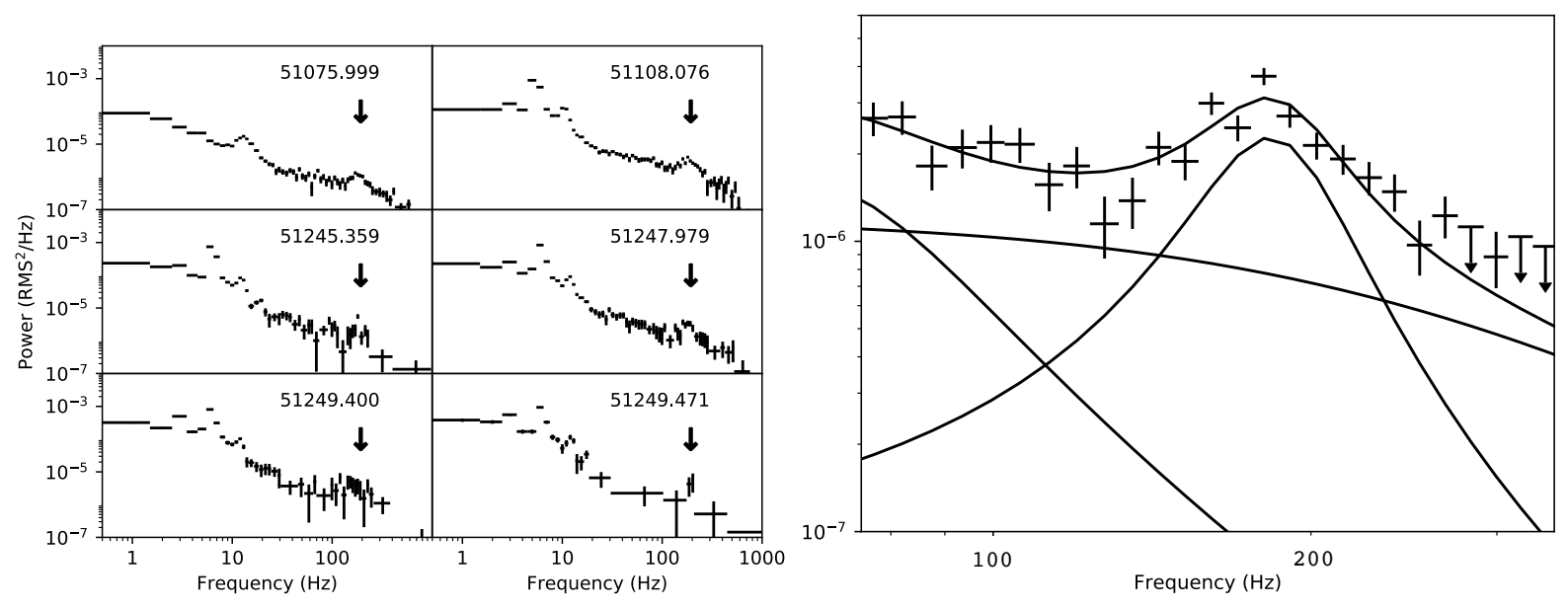

Fig. 3.- Left: Individual rebinned PDSs of the six observations of group II with a potential $183 \mathrm{~Hz}$ HFQPO. The vertical arrow shows the approximate location of the HFQPO, which, in all cases, is consistent with an excess. Right: Zoom on the combined PDS from the six observations. The continuous lines represent the best fit to the 50-1000 Hz range, and the individual components of the fit.

advantage of the large photon flux of the MJD 51076.0 observation ${ }^{3}$. The HFQPO is visible in at least three of the individual observations and is very obvious in the the cumulative PDS (Fig.3). The 50-1000 Hz range of the cumulative PDS is best fitted by a model comprised of two broad Lorentzians (one zero centered and one centered at about $60 \mathrm{~Hz}$ ), and a thinner feature, a HFQPO, at $183 \mathrm{~Hz}$. Although the fit is not perfect $\left(\chi_{\nu}^{2}=1.56\right.$ for 33 dof) attempts to include additional thin features at any frequency failed with estimated upper limits of about $0.8 \%$ for a putative $Q=10$ HFQPO. Of all HFQPOs detected in this study it is the one with the lowest quality factor $(\mathrm{Q} \sim 2.9)$. We also tested the hypothesis that this broad feature is due to a blend of two narrower peaks, but did not manage to obtain satisfactory fits when replacing the broad Lorentzian by two narrow ones even when

\footnotetext{
${ }^{3}$ We also considered an averaged PDS obtained while omitting the observation of MJD 51076.0, but we obtained no significant differences for the parameters of the combined PDS.
} 
fixing some of their parameters.

\subsection{Group III: HFQPO at $\sim 250 \mathrm{~Hz}$}

Figure 4 shows the PDSs of all the observations considered for the analysis of the $\sim 250$ $\mathrm{Hz}$ feature. The $250 \mathrm{~Hz}$ QPO was reported both during the 1998-99 and 2000 outbursts (Remillard et al. 2002b; Miller et al. 2001). It is visible in most of the observations considered here. All individual PDSs are rather similar with a low frequency flat top noise and a LFQPO at $\sim 9 \mathrm{~Hz}$ (see also Sobczak et al. 2000b; Rodriguez et al. 2004). The LFQPO is reported as a potential type $\mathrm{A}$ with a certain caution in the classification (Remillard et al. 2002b) during the 1998-99 outburst, and mentioned as type B during the 2000 one (Rodriguez et al. 2004).

When omitting the frequencies below $50 \mathrm{~Hz}$, the continuum of the combined PDS is rather well represented with a broad zero-centered Lorentzian, although with large residuals in the frequency range of the previously reported QPOs (Remillard et al. 2002b; Miller et al. 2001; Belloni et al. 2012). A thin additional Lorentzian $\left(\nu_{\mathrm{QPO}}=245_{-15}^{+22} \mathrm{~Hz}\right)$, provides a good fit $\left(\chi_{\nu}^{2}=1.2,26\right.$ dof $)$, to the data but the width of the feature tends to very high values that are not compatible with a QPO. A good fit is obtained $\left(\chi_{\nu}^{2}=1.16,22\right.$ dof $)$ when fixing the width of the feature to $50 \mathrm{~Hz}$ (close to the widths reported in Miller et al. (2001)). In this case the best value found for the QPO frequency is about $260 \mathrm{~Hz}$ (Table 1 ).

In an attempt to verify if the tendency of the fit to converge toward a broad feature was not due to the presence of two HFQPOs, and since in the fixed width fit some residuals are still visible at about $200 \mathrm{~Hz}$, we, in a second run included a second thin Lorentzian to the model. The fit quality marginally improved $\left(\Delta \chi^{2}=13\right.$ for 3 additional dof $)$, with a final $\chi_{\nu}^{2}<1$ indicating that the model oversamples the PDS. The width of the second component is unconstrained, and fixing it to $50 \mathrm{~Hz}$ permits a good fit to be obtained with a $\chi_{\nu}^{2}$ close to 
one (25 dof). The statistical significance of the second feature is $3.1 \sigma$. Given this rather low significance, and the marginal improvement brought by the inclusion of this second putative feature, one can question its genuineness, and we prefer to consider it as a possible second QPO rather than a soundly confirmed one. Bearing this in mind, the ratio of the frequencies of the two HFQPOs $\left(f_{\text {low }} / f_{\text {high }}\right)$ would then be $0.75_{-0.03}^{+0.02}$. It is interesting to remark that the centroid frequency of the main QPO $(265 \mathrm{~Hz})$ is almost exactly the value of the 'hard' $263 \mathrm{~Hz}$ QPO reported in two occasions by Miller et al. (2001) and Belloni et al. (2012) from a hard band only.
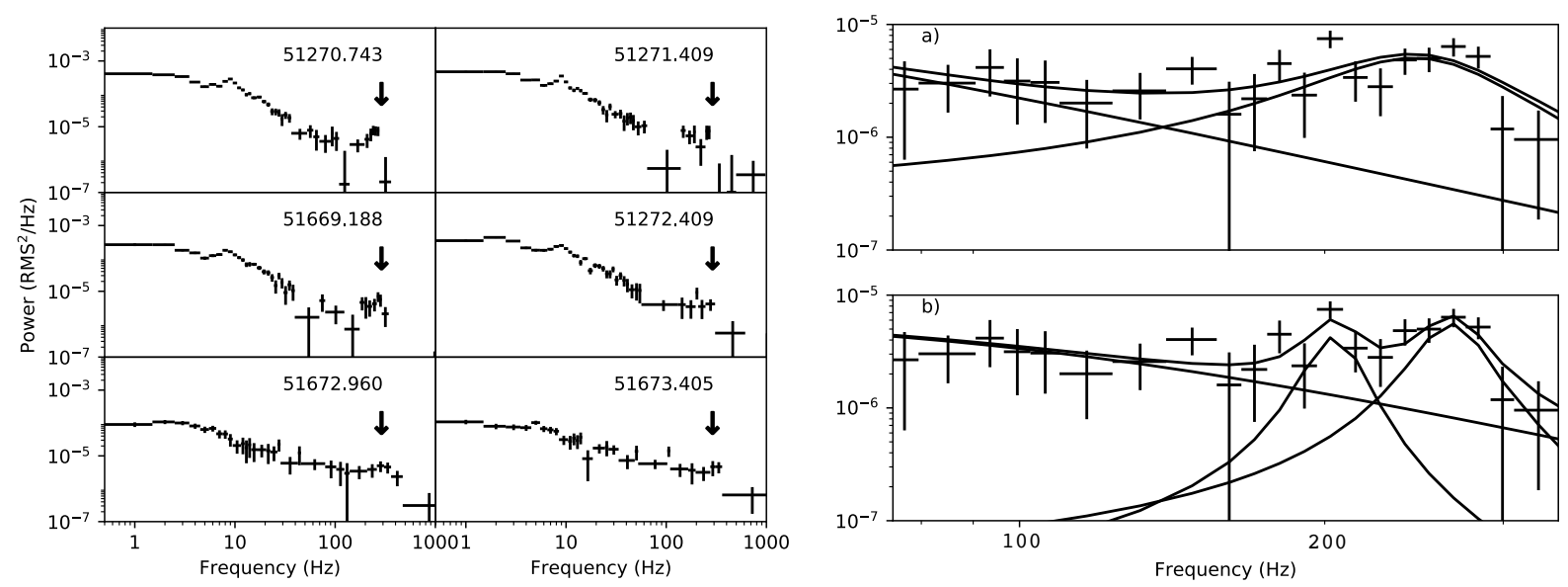

Fig. 4.- Left: Individual rebinned PDSs of the six observations of group III considered for the analysis of the $250 \mathrm{~Hz}$ QPO. The vertical arrow shows the approximate location of the HFQPO, which is, in most cases, consistent with a slight excess.Right: Zoom on the combined PDS from the seven observations with the best fitted model. a) With a single broad QPO at $\sim 245 \mathrm{~Hz}$. b) With a pair of HFQPOs. The continuous lines represent the best fit model and the individual components of the fit.

In order to check that this occurrence of a potential pair of HFQPO was not due to a mixing of observations with different QPOs (although none other than the $250 \mathrm{~Hz}$ were

\footnotetext{
${ }^{4}$ Dates are reported with a one day error in this paper
} 
previously reported from the observations considered in this group), we produced a PDS from the three observations where the feature was previously reported (Miller et al. 2001; Remillard et al. 2002b, Table 2.1). The resulting PDS (not shown) is well fitted (above $50 \mathrm{~Hz}$ ) by a broad zero-centered Lorentzian plus a thin one $\left(\chi_{\nu}^{2}=0.9\right.$ for 23 dof $)$. The width is however unconstrained and the centroid found at $244\left({ }_{-17}^{+14}\right) \mathrm{Hz}$. We then froze the width to $50 \mathrm{~Hz}$ (Miller et al. 2001), and obtained a satisfactory fit ( $\chi_{\nu}^{2}=0.9,24$ dof) with

$\nu_{\mathrm{QPO}}=250 \pm 11 \mathrm{~Hz}$, and $\mathrm{P}=2.2_{-0.4}^{+0.3} \% \mathrm{RMS}$. It is significant at $5.1 \sigma$. We estimate a $3 \sigma$ limit of about $1.5 \%$ (resp. $1.8 \%$ ) for the presence of an additional $Q=10$ (resp. $Q=4$ ) feature at $200 \mathrm{~Hz}$.

Similarly, we then considered a PDS averaged over the three observations where no QPO was previously reported (Table 2.1). The PDS is well represented by a single broad zero-centered Lorentzian $\left(\chi_{\nu}^{2}=0.9\right.$ for 24 dof $)$. We estimate a $3 \sigma$ limit of about $1.9-2.0 \%$ for a $50 \mathrm{~Hz}$ FWHM HFQPO at 200-260 Hz.

\subsection{Group IV: HFQPO at $\sim 275 \mathrm{~Hz}$}

Figure 5 shows the PDSs of all the observations considered for the analysis of the $\sim 275 \mathrm{~Hz}$ feature. As for the $250 \mathrm{~Hz}$, the HFQPO near $275 \mathrm{~Hz}$ was reported in both the 1998-99 and 2000 outbursts (Remillard et al. 2002b; Miller et al. 2001; Belloni et al. 2012). All individual PDSs are roughly similar, with a flat top noise component and a broad low frequency feature (at about $5.5 \mathrm{~Hz}$ ) on top of it with a potential harmonic around 10 $\mathrm{Hz}$ (see Sobczak et al. 2000b; Rodriguez et al. 2004). The LFQPO is reported as a type A in the MJD 51115.28 observation and a possible type A with a certain caution in the classification in the MJD 51258 observations by Remillard et al. (2002b).

When omitting the frequencies below $50 \mathrm{~Hz}$, the combined PDS is rather well represented $\left(\chi_{\nu}^{2}=1.3\right.$ for 17 dof $)$ with a broken power law continuum (with a rather flat 
first component and a break at about $230 \mathrm{~Hz}$ ) and an additional thin feature at $\sim 275 \mathrm{~Hz}$ (Fig. 5 right, Table 1). This model is preferred to a broad zero-centered Lorentzian plus the QPO which gives a poorer fit $\left(\chi_{\nu}^{2}=1.6\right.$ for 19 dof $)$ or just a broad Lorentzian plus the QPO $\left(\chi_{\nu}^{2}=1.5\right.$ for 18 dof $)$. As the model of the underlying continuum influences the results of the QPO we nevertheless estimated the parameters of the QPO with the three models. In all cases, the frequencies obtained are the same, with similar errors, the width is slightly lower in the broken powerlaw case even if all widths are consistent within the large errors, and the RMS amplitude is also slightly higher in the zero-centered case $(1.5 \pm 0.2 \%)$ than in the broken power law fit $(1.2 \pm 0.3 \%$, Table 1$)$, although all values are compatible within the $90 \%$ errors. We thus consider in the following the broken powerlaw results only. This HFQPO is significant at about $6 \sigma$. No other peak is obvious in the combined PDS and we estimate $3 \sigma$ upper limits comprised between $\sim 1 \%$ and $1.8 \%$ for the presence of an additional HFQPO in the considered range.
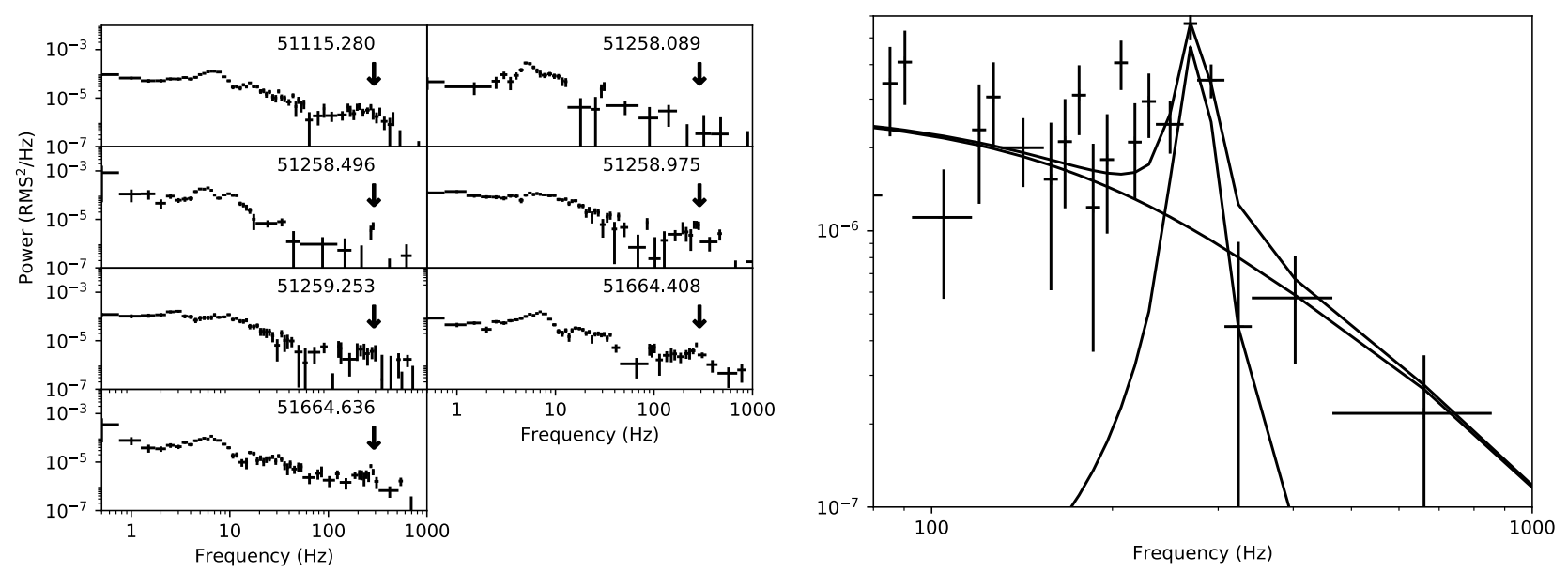

Fig. 5.- Left: Individual rebinned PDSs of the six observations of group IV with a potential $275 \mathrm{~Hz}$ HFQPO. The vertical arrow shows the approximate location of the HFQPO, which is, in most cases, consistent with a slight excess.Right: Zoom on the $80-1000 \mathrm{~Hz}$ region of the combined PDS from the six observations. The continuous lines represent the best fit model and the individual components of the fit. 


\subsection{Group V: HFQPO at $\sim 283 \mathrm{~Hz}$}

Figure 6 shows the PDSs of all the observations considered for the analysis of the $\sim 283$ $\mathrm{Hz}$ feature. When omitting the frequencies below $50 \mathrm{~Hz}$, the continuum of the combined PDS is well modeled by one broad zero centered Lorentzian. This combined PDS clearly shows the presence of a peak at $\sim 280 \mathrm{~Hz}$, and adding a thin Lorentzian provides a rather good fit $\left(\chi_{\nu}^{2}=1.5\right.$ for 21 dof $)$ to the PDS, but large residuals still remain. The inclusion of a second narrow Lorentzian slightly improves the fit $\left(\chi_{\nu}^{2}=1.2\right.$ for 18 dof, ie $\Delta \chi^{2}=10$ for 3 additional degrees of freedom). The width of this extra component is, however, not constrained, and tends to very high value especially when the continuum is not fixed. This effect is clearly due both to the restrained frequency range of our fit, and the three bins around $60-80 \mathrm{~Hz}$ that tend, here, to be considered as a thin feature (while there are not when considering the broad band PDS). In a second pass we froze its width to the centroid of the best value obtained when left free (Table 1). In both the fits with or without the second peak, the $283 \mathrm{~Hz}$ QPO is significant at $7 \sigma$. We estimate the significance of the second feature to be just at the $3 \sigma$ level (Table 1). Here again, given the rather low significance of the second potential QPO, and the marginal improvement brought by the inclusion of this second putative feature, we prefer to consider it as a possible second QPO rather than a confirmed one. Bearing this in mind, the ratio of the frequencies of the two $\operatorname{HFQPOs}\left(f_{\text {low }} / f_{\text {high }}\right)$ would then be $f_{\text {low }} / f_{\text {high }} \sim 0.72_{-0.07}^{+0.04}$ 

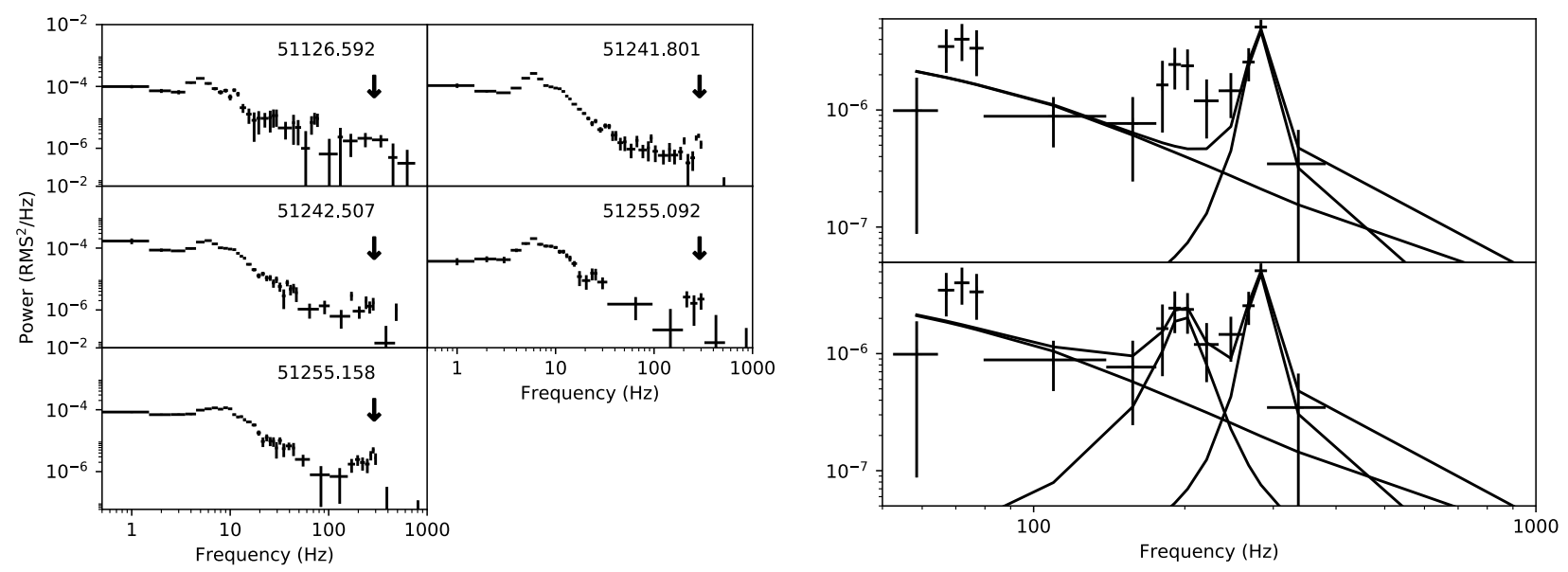

Fig. 6.- Left: Individual rebinned PDSs of the five observations of group V considered for the analysis of the $283 \mathrm{~Hz}$ QPO. The vertical arrow shows the approximate location of the HFQPO, which is, in most cases, consistent with a slight excess. Right: Zoom on the 50-1000 Hz range of the combined PDS from the five observations with the best fitted model, that includes a pair of HFQPOs. The continuous lines represent the individual components of the fit. 
Table 1: Parameters of the HF QPOs obtained from the fits to the stacked PDSs of each group of data. The errors correspond to the $90 \%$ level of uncertainty.

\begin{tabular}{lcccc} 
Group & $\begin{array}{c}\text { HFQPO } \\
\text { freq }(\mathrm{Hz})\end{array}$ & $\begin{array}{c}\text { FWHM } \\
(\mathrm{Hz})\end{array}$ & $\begin{array}{c}\text { Power } \\
(\% \mathrm{RMS})\end{array}$ & significance $^{a}$ \\
\hline $\mathrm{I}(\sim 140 \mathrm{~Hz})$ & $143 \pm 4$ & $16.5^{c}$ & $0.9 \pm 0.2$ & $4.2 \sigma$ \\
\hline $\mathrm{II}(\sim 183 \mathrm{~Hz})$ & $182 \pm 4$ & $64_{-21}^{+24}$ & $1.5 \pm 0.3$ & $11 \sigma$ \\
\hline $\mathrm{III}^{d}(\sim 250 \mathrm{~Hz})$ & $260_{-11}^{+10}$ & $50^{c}$ & $1.9 \pm 0.3$ & $\sim 5 \sigma$ \\
& -------------------- & $\sim 5.9 \sigma$ \\
$\mathrm{III}{ }^{e}(\sim 250 \mathrm{~Hz})$ & $267 \pm 8$ & $50^{c}$ & $2.0 \pm 0.3$ & $3.1 \sigma$ \\
& $200_{-13}^{+11}$ & 50 frozen & $1.6_{-0.5}^{+0.3}$ & $5.9 \sigma$ \\
\hline $\mathrm{IV}(\sim 275 \mathrm{~Hz})$ & $276_{-4}^{+2}$ & $10_{-8}^{+25}$ & $1.2 \pm 0.3$ & $\sim 7 \sigma$ \\
\hline $\mathrm{V}(\sim 283 \mathrm{~Hz})$ & $280_{-4}^{+5}$ & $19_{-13}^{+15}$ & $1.3 \pm 0.2$ & $\sim 3 \sigma$
\end{tabular}

a Significance calculated for the best fitted, or for a fixed width.

${ }^{b}$ Parameter pegged at the upper limit for the feature to be considered a QPO.

c Parameter frozen to the common value found in the literature.

$d$ Group III fitted with only one broad HFQPO (Fig. 4 a).

e Group III fitted with two HFQPOs (Fig. $4 \mathrm{~b})$.

\section{Discussion}

\subsection{Is the lack of multiple peaks due to the limited statistics of some PDSs?}

Our first aim here was to check if multiple peaks are present but not detected because they are below the detection level in the single observations. Our definition of the groups 
permitted us to significantly extend the total duration of each considered sample, and thus lower the level of detectability for thin features in the combined PDSs (Table 2.1) except for group I. In our five groups we get two potential ( $3 \sigma$ level) pair detection in group III and group V (Table 1), while the very short exposure $(3.5 \mathrm{ks})$ of group I does not permit to establish the reality of the $1.7 \sigma$ peak mentioned above. The absence of detection of any additional peak(s) (other than those already reported in the literature) in groups II and IV, clearly indicated that increased statistics does not always lead to the detection of multiple peaks. Our best example is group II which has the longest total exposure, of about $18 \mathrm{ks}$, but shows only one broad peak with a $3 \sigma$ fractional RMS limits of $0.8 \%$ for the presence of an additional HFQPO.

This in turn raises another question. What are the conditions that trigger the presence, or more precisely the detectability, of more than one peak? While fully answering this question is beyond the scope of this paper we can look at some directions for future studies. Interestingly, while group IV and V have rather similar PDSs, one shows a potential pair of peaks and the other does not. The fact that group IV has a HFQPO similar to that of group V, but no other peak detected suggests that other parameters have an impact on the number of significant HF features in the PDSs. They could, for example, be influenced by the overall continuum shape and power, or the full LFQPO structure, that shows, here, strong differences as we briefly mention here in the introduction of each group, and as is obvious from the in-depth analysis of the 1998-1999 and 2000 outbursts (Remillard et al. 2002b; Rodriguez et al. 2004).

Another possibility is a difference of the spectral behavior of the source between the different groups. While a spectral analysis of the five groups we defined is not available and beyond the scope of this paper, we can look at the source behavior during the individual 


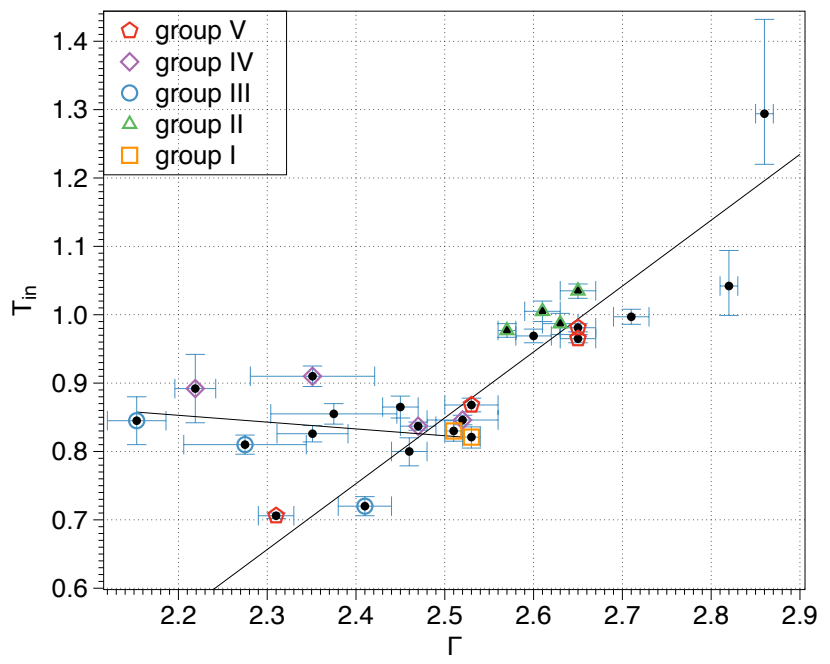

Fig. 7.- Representation of the behavior of the inner temperature $T_{i n}$ versus the Photon index $\Gamma$ for all the HFQPOs detected in the 98-99 and 2000 outburst as in Miller et al. (2001); Remillard et al. (2002a). Group I is represented by the hollow orange square, group II the hollow green triangle, group III the hollow blue circle, group IV the hollow purple diamond and group $\mathrm{V}$ the hollow red pentagon. 
observations. Direct comparison between the HFQPO frequency and the power law photon index $(\Gamma)$ of the energy spectra (Sobczak et al. 2000a; Rodriguez et al. 2003) does not show any particular relation but groupe IV has a power law photon index between 2.22 and $2.52 \pm 0.04$ while group $\mathrm{V}$ is slightly higher with $\Gamma$ between 2.31 and $2.65 \pm 0.02$. Similarly, group V exhibits a wider range of inner temperature $\left(T_{\text {in }}\right.$ between 0.71 and 0.98 \pm 0.006 ) than group IV (between 0.837 and $0.910 \pm 0.015$ ). When putting those together the behavior of group IV and $\mathrm{V}$ in the $\left(\Gamma, T_{i n}\right)$ space is perpendicular to one-another (see Fig 7). Altogether, this shows that both groups have a different spectral behavior which might be the cause of only one group having a second detectable peak.

\subsection{Status of the 3:2 ratio in our dataset}

Now that we have shown that stacking together PDSs of similar HFQPO observations, can sometimes lead to a second, tenuous $(3 \sigma)$, peak detection, we can study how the detected frequencies relate to one another. In group $\mathrm{V}$ we have a peak ratio $\nu_{1, \mathrm{HF}} / \nu_{2, \mathrm{HF}}=0.72_{-0.07}^{+0.04}$ which is compatible with several integer ratios, thus closer to being $3: 4$, while the $2: 3$ ratio is at the boundary of the errors. If we look at group III the ratio of observed frequencies is $\nu_{1, \mathrm{HF}} / \nu_{2, \mathrm{HF}}=0.75_{-0.03}^{+0.02}$ which is close to the ratio $3: 4$ but incompatible with a $2: 3$. So while the potentially detected pairs of frequencies could be in some integer relationship with one another, we did not detect a firm 2:3 ratio between the peaks but, we instead obtained preferably higher integer ratios in the two cases where we have detected a possible pair of HFQPOs. We can therefore simply conclude that the occurrence of HFQPOs in pairs with

specific or preferred frequency ratio (in particular the 3:2) should not be taken as a strong requirement for HFQPO models. 
Let us now now consider the possibility that all the (HFQPO) frequencies exhibited by XTE J1550-564 relate to one another in an integer ratio (ie that they are somehow all harmonically related). While this assumption may first appear counter-intuitive there are several facts pointing in that direction. First of all, the fact that the detected frequency can be relatively similar for observations that are separated by years (e.g. Belloni \& Altamirano 2013), points toward a mechanism able to trigger the same frequencies at very different, non connected moments (in particular different outbursts). Second the, now, potentially detected simultaneous occurrence of pairs, indicates a mechanism able to stimulate multiple frequencies at the same time. So, assuming that all the XTE J1550-564 HFQPOs are indeed a manifestation of the same mechanism we can associate the HFQPO frequency of the different groups to harmonic numbers. In this exercise, one would need to allow for a much lower base/fundamental frequency and much higher ratio and number of harmonics, to fully represent the whole sample. For example, the detection of HFQPOs at $141 \mathrm{~Hz}, 198$ $\mathrm{Hz}$, or $281 \mathrm{~Hz}$, would then require to go up to mode 9 or 10 with a fundamental frequency around 30-35 Hz.

While this cannot be totally excluded, it rather raises extra difficulties and questions regarding, for example, the main selection criteria for these different modes. While we do not favor this interpretation, it is interesting to note that such a high number of modes is sometimes seen in fully General Relativistic disk simulations Casse et al. 2017, Casse \& Varnière 2018, submitted) but rarely in the Pseudo-Newtonian case (Vincent et al. 2013).

Another way to reconcile those observations with the peaks being harmonically related without the need to have high modes is to relax the assumption that the frequency of the fundamental is mostly constant. So, depending on the physical condition in the system, we keep a relationship between the peaks up to four with a base frequency that can vary by a factor of up to two. This interpretation, however, has its own set of difficulties, mainly 
related to the origin of the limited variation of this base frequency.

Another possibility is that our limited sample is not representative of the full extent of the variation of the HFQPO frequency, or the set of frequencies are related to a single peak, in which cases the models need to incorporate an ever larger range of variation.

In conclusion, by increasing the total exposure of five HFQPOs' frequencies, we did not

detect another peak for three groups, while also obtaining two $3-\sigma$ potential detections of a second peak, neither of which favored a 3:2 ratio.

\subsection{Constraints for HFQPO models}

Using the known facts and the results obtained here one can form a list of contraints that any HFQPO model needs to explain.

- an HFQPO model needs to modulate the X-ray flux.

This is of course an obvious fact, and might be the most straightforward constraint but it is not often addressed by the theoretical models.

- A single mechanism should be able to predict the distribution of frequencies.

The distribution of frequencies shows some dispersion. Models, therefore, need to be able to explain the origin of this distribution and on what it depends.

- The model should be able to explain the HFQPO frequencies' selection mechanism: We have seen that HFQPOs appear in pairs or alone. Models, therefore, need to be able to explain the selection mechanism that favours the occurrence of one of the 
HFQPOs instead of the other, and the appearance of pairs in some cases and single peaks in others. This is particularly obvious from the comparison of group IV and V that show relatively similar continuums but different QPO complexes at high frequency.

- an HFQPO model should be able to exist in a wide variety of initial conditions. Indeed, strong LFQPOs are often observed in the presence of HFQPOs, therefore it is required to have a regime where both models exist simultaneously in the system. This is especially important as LFQPOs tend to have a much stronger influence on the system and will change the state/initial condition in which the HFQPO model is developing.

\section{Summary}

We created five groups of combined PDSs by adding together observations having similar HFQPOs, continuums, and LFQPOs from the 1998-99 and 2000 outbursts of XTE J1550-564. The HFQPO frequencies span from 140 to $283 \mathrm{~Hz}$ respectively from group I to V. The improved statistics of the combined PDSs leads to two probable detections of a pair of HFQPOs in group III and group V.

The most striking feature of those pairs is that their frequency ratios are not in the previously detected 3:2 ratio even though we used some of the observations in which they were previously detected. Instead the frequency ratio in these two cases is closer to 0.75 . If one assumes that the two peaks are harmonically related, then the features would need to be the third and fourth harmonics of a yet to be detected fundamental at a much lower frequency. Because we only added together observations having similar originally detected frequencies and continuum, this means that the 3:2 ratio is not always present in XTE J1550-564. In turn, theoretical models need to focus on explaining the frequency selection 
mechanism which gives two 3:4 HFQPOs in some cases, and also why a pair is present in group $\mathrm{V}$ and not in group IV, while the two PDSs do show very similar behavior.

The authors thank the anonymous referee that helped to clarify several points an to improve the paper to this final form. We acknowledge the financial support of the UnivEarthS Labex program at Sorbonne Paris Cite (ANR-10-LABX-0023 and ANR-11IDEX-0005-02). JR acknowledges funding support from the French Research National Agency: CHAOS project ANR-12-BS05-0009 (http://www . chaos-project.fr). 


\section{REFERENCES}

Altamirano, D., \& Belloni, T. 2012, ApJ, 747, L4

Belloni, T., Méndez, M., \& Sánchez-Fernández, C. 2001, A\&A, 372, 551

Belloni, T. M., \& Altamirano, D. 2013, MNRAS, 432, 19

Belloni, T. M., Sanna, A., \& Méndez, M. 2012, MNRAS, 426, 1701

Casse, F., Varniere, P., \& Meliani, Z. 2017, MNRAS, 464, 3704

Miller, J. M., Wijnands, R., Homan, J., et al. 2001, ApJ, 563, 928

Remillard, R. A., \& McClintock, J. E. 2006, ARA\&A, 44, 49

Remillard, R. A., Muno, M. P., McClintock, J. E., \& Orosz, J. A. 2002a, ApJ, 580, 1030

Remillard, R. A., Sobczak, G. J., Muno, M. P., \& McClintock, J. E. 2002b, ApJ, 564, 962

Rodriguez, J., Corbel, S., Kalemci, E., Tomsick, J. A., \& Tagger, M. 2004, ApJ, 612, 1018

Rodriguez, J., Corbel, S., \& Tomsick, J. A. 2003, ApJ, 595, 1032

Sobczak, G. J., McClintock, J. E., Remillard, R. A., et al. 2000a, ApJ, 544, 993

-. 2000b, ApJ, 531, 537

Strohmayer, T. E. 2001, ApJ, 554, L169

Varnière, P., Tagger, M., \& Rodriguez, J. 2011, A\&A, 525, A87+

-. 2012, A\&A, 545, A40

Vincent, F. H., Meheut, H., Varniere, P., \& Paumard, T. 2013, A\&A, 551, A54 